\title{
Dynamics of a protein and its surrounding environment: A quasielastic neutron scattering study of myoglobin in water and glycerol mixtures
}

\author{
H. Jansson, ${ }^{1,2, a)}$ F. Kargl, ${ }^{1,3}{ }^{1,}$ F. Fernandez-Alonso, ${ }^{4}$ and J. Swenson ${ }^{1}$ \\ ${ }_{1}^{1}$ Department of Applied Physics, Chalmers University of Technology, SE-41296 Göteborg, Sweden \\ ${ }^{2}$ The Swedish NMR-Centre, Göteborg University, SE-405 30 Göteborg, Sweden \\ ${ }^{3}$ Institute of Mathematics and Physics, Aberystwyth University, Aberystwyth SY23 3BZ, \\ United Kingdom \\ ${ }^{4}$ ISIS Facility, Rutherford Appleton Laboratory, Chilton, Didcot Oxfordshire OX11 OQX, \\ United Kingdom
}

(Received 9 December 2008; accepted 28 April 2009; published online 27 May 2009)

In this quasielastic neutron scattering (QENS) study we have investigated the relation between protein and solvent dynamics. Myoglobin in different water:glycerol mixtures has been studied in the temperature range of 260-320 K. In order to distinguish between solvent and protein dynamics we have measured protonated as well as partly deuterated samples. As commonly observed for bulk as well as for confined water, the dynamics of the surrounding solvent is well described by a jump diffusion model. The intermediate scattering function $I(Q, t)$ from the protein (partly deuterated samples) was analyzed by fitting a single Kohlrausch-Williams-Watts (KWW) stretched exponential function to the data. However, due to the limited experimental time window, two different curve fitting approaches were used. The first one was performed with the assumption that $I(Q, t)$ decays to zero at long times, i.e., it was assumed that all protein relaxations that are observed on the experimental time scale, as well as would be observed on longer time scales, can be described by a single KWW function. In the second approach we instead assumed that both the protein relaxation time $\tau_{p}$ and the stretching parameter $\beta_{\mathrm{Kww}}$ were $Q$-independent, i.e., we assumed that the protein dynamics is dominated by more local motions. Advantages and disadvantages of both approaches are discussed. The first approach appears to work best at higher $Q$-values, indicating a power law relation of the $Q$-dependent protein dynamics for all samples and temperatures, whereas the second approach seems to work at lower $Q$-values, where the expected confined diffusion of hydrogen atoms in the protein gives the assumed $Q$-independent relaxation time. Independent of the chosen approach we find a significant correlation between the average relaxation time of the protein and the diffusion constant (or in this case the related relaxation time) of the solvent. However, the correlation is not perfect since the average relaxation time of the protein is more strongly dependent on the total amount of solvent than the diffusion constant of the solvent itself. Thus, the average relaxation time of the protein decreases not only with increasing solvent mobility, but also with increasing solvent content. (C) 2009 American Institute of Physics. [DOI: 10.1063/1.3138765]

\section{INTRODUCTION}

The presence of water is essential for structure, dynamics, and functions of biomolecules. The amount of water in the system has a strong effect on the biomolecular dynamics and on their specific biological role. For instance, it has been shown for a number of proteins that their specific function is lost in the dehydrated state, and that the activity increases rapidly when the water content reaches a characteristic hydration level, see, e.g., Refs. 1-5. The reason for this is that without water a protein shows no biologically important dynamics, and as a result, it cannot perform any biological activities. ${ }^{6,7}$ The water molecules surrounding a protein strongly interact with the protein surface by forming hydrogen bonds to the main chain or the side chain functional groups of the protein. The consequence of this interaction is that the motion of the water molecules close to the protein

${ }^{a)}$ Electronic mail: helen.jansson@ chalmer.se. surface is restricted and differs thereby from bulk water [the translational diffusion may slow down by as much as a factor 10 (Refs. 8 and 9)]. The retarded water motion is, however, of great biological importance since it enables protein motions and proton transfer along the protein surface, which are both necessary for biological processes. ${ }^{10}$ Thus, water is known to be of fundamental importance for proteins, but how and why a protein is affected by its surrounding is poorly understood and currently a matter of active research and discussion, see, e.g., Refs. 11-16.

A technique frequently used for studies of structure and dynamics of proteins as well as the relation between protein dynamics and motions in its surroundings is neutron scattering since it is, in principle, possible with this technique to distinguish between the protein and its surrounding by use of isotopic substitution. Most of the neutron scattering studies presented in literature related to protein dynamics and the dynamics of its surrounding environment focus on the socalled dynamical transition (see, e.g., Ref. 17-25), i.e., the 
temperature region where there is an onset of anharmonic motions on the experimental time scale, commonly identified by a change in slope in the temperature dependence of the elastic intensity or the mean square displacement (MSD) when heating the sample from low temperatures. In some of those studies ${ }^{19,24,25}$ the authors investigated how and why the water molecules in the vicinity of a protein surface influence the dynamics of the protein by comparing how different types of solvents influence the protein dynamics and activity. From investigations, including both experimental and simulation studies, it has been concluded that the onset of anharmonic protein motions on a certain experimental dynamical time scale is solvent dependent and occurs both in water and glycerol but not in dry proteins. ${ }^{26,27}$ This onset should not be confused with an onset related to the rotation of protein methyl groups that independent of the solvent level generally occurs at a lower temperature. ${ }^{23}$ In the case of proteins dissolved in water the so-called dynamical transition is located around $220 \mathrm{~K}$ whereas for proteins in glycerol such an onset is visible at about $280 \mathrm{~K}$. The reason for this large difference in onset temperature is that the addition of water to the protein enhances the flexibility of the protein structure whereas glycerol acts as a stabilizer, which results in a decreased structural flexibility as compared to the structural flexibility in water at a similar solvent level. However, so far, the microscopic origin of the rapid increase in MSD of proteins at a certain temperature remains unclear and many suggestions have been made. For instance, from studies that combine neutron scattering and molecular dynamics (MD)-simulations ${ }^{14,27}$ it has been suggested that the dynamical transition is related to the relaxation of the proteinsolvent hydrogen network, which, in turn, is connected to the onset of translational diffusion of solvent molecules. In other studies $^{17,28,29}$ the authors pointed out that the dynamical transition is not associated with any rapid or anomalous change of the protein dynamics. Rather, it is simply a result of that the dynamics of the protein becomes faster than the maximum time scale that is resolved by the spectrometer. As a consequence, this means that the temperature where this socalled dynamical transition occurs depends on the experimental energy resolution (i.e., the longest time scales that can be probed by the instrument).

Despite the fact that the protein function is closely related to its conformation and dynamics, ${ }^{30,31}$ and regardless of that it has been suggested that different types of solvent motions control different types of protein motions, ${ }^{11}$ only a minority of the neutron scattering studies concerning protein dynamics found in literature have been performed with the aim to explore the nature of the dynamics. The results from some of those studies (see, e.g., Refs. 32-34) imply that the protein dynamics can be described by confined diffusion whereas in one study ${ }^{35}$ the authors suggest that the dynamical behavior can be divided into several dynamical regions (depending on the spatial length scale, i.e., the $Q$-range) in a similar way as for polymers. Moreover, in another study ${ }^{36}$ in which the growth of protein internal dynamics with increasing water content was investigated, it was concluded that a local diffusivity of the side chains located at the protein surface was progressively enhanced when adding up to about a monolayer of water to a dry protein powder, and that a further increase in the water content enhances the rate of these diffusional motions. In this study on myoglobin in waterglycerol mixtures of different water contents, we have explored the possible relation between protein and solvent dynamics and tried to investigate the physical nature of the dynamics of both the protein and its surrounding solvent.

\section{EXPERIMENTAL}

In a neutron scattering experiment the dynamic structure factor $S(Q, \omega)$ is measured. The total scattering contains information about both coherent and incoherent scattering, which means that $S(Q, \omega)$ measures interparticle as well as self-correlations in space and time, respectively. The main contribution to the total scattering in biological materials is incoherent scattering due to the large incoherent scattering cross section of hydrogen compared to the total scattering cross section of other elements. Since the scattering cross section is not only different for different elements but also different for different isotopes, such as $\mathrm{H}$ and $\mathrm{D}$, it is possible to distinguish between motions of proteins and its surrounding environment by use of H/D isotope substitution.

For this study horse heart myoglobin (Sigma-Aldrich, product number M1882) was chosen. The protein was in form of a freeze-dried powder and used without any further treatment. Note here that the "dry" protein powder contains a small amount of bound water $(\leq 10$ wt $\%)$. For the measurements isotopic substitution was used for this globular protein, i.e., both protonated and partly deuterated samples were prepared, see Table I. The solvents used were water and water:glycerol mixtures of ratios 25:75 and 50:50 wt \%. Table I provides detailed information about the solvent levels ( $h$ $=\mathrm{g}$ solvent $/ \mathrm{g}$ protein), number of solvent molecules per protein molecule for the different samples, and measured temperatures. It should be noted here that there is a difference of about $10 \%$ in the total number of solvent molecules per protein molecule between protonated and deuterated samples. Partly deuterated protein powder was obtained by dissolving the protein in $\mathrm{D}_{2} \mathrm{O}$ and subsequently freeze-drying it. This process was repeated two times in order to remove as many of the exchangeable protons, mostly located at the protein surface, as possible. The desired solvent level was obtained by mixing the dry protein with the appropriate amount of its solvent. For the protonated samples doubly distilled water (Milli Q-water) and glycerol (Sigma-Aldrich) were used. The deuterated samples were prepared with $\mathrm{D}_{2} \mathrm{O}$ and deuterated glycerol $\left(\mathrm{C}_{3} \mathrm{D}_{8} \mathrm{O}_{3}\right)$ from Larodan Fine Chemicals as solvents.

The QENS measurements were carried out at a number of different temperatures, see Table I, on the high-resolution inverse-geometry backscattering spectrometer IRIS at the pulsed neutron spallation source ISIS at the Rutherford Appleton Laboratory, U.K. The IRIS spectrometer is described in detail in Ref. 37, so here we will only give some specific details for the present measurements. Using the PG002 analyzers and an incident neutron wavelength of about $6.6 \AA$, an energy resolution of $17.5 \mu \mathrm{eV}$ [full width at half maximum (FWHM)] and a total energy window of 
TABLE I. Samples, solvent compositions, and measured temperatures. For all samples containing $\mathrm{D}_{2} \mathrm{O}$, the proteins are partly deuterated (as described in the text), and the glycerol is fully deuterated and denoted d-gly in the table. As seen from the table, the difference between the number of solvent molecules per protein molecule is about $10 \%$ for the protonated compared to the deuterated samples.

\begin{tabular}{lccc}
\hline \hline Sample & $\begin{array}{c}\text { Solvent level } \\
h\end{array}$ & $\begin{array}{c}\text { Solvent molecules } \\
\text { per protein molecule }\end{array}$ & $\begin{array}{c}\text { Measured } \\
\text { temperatures (K) }\end{array}$ \\
\hline $\mathrm{Mb} / \mathrm{H}_{2} \mathrm{O}$ & $h=0.5$ & 464 & $260,280,310$ \\
$\mathrm{Mb} / \mathrm{D}_{2} \mathrm{O}$ & & 418 & \\
$\mathrm{Mb} / \mathrm{H}_{2} \mathrm{O}$ & $h=1.0$ & 928 & $260,280,300,320$ \\
$\mathrm{Mb} / \mathrm{D}_{2} \mathrm{O}$ & & 835 & 290 \\
$\mathrm{Mb} / \mathrm{H}_{2} \mathrm{O}:$ gly $25: 75 \mathrm{wt} \%$ & $h=2$ & 454 & \\
$\mathrm{Mb} / \mathrm{D}_{2} \mathrm{O}:$ d-gly $25: 75$ wt $\%$ & & 418 & $260,280,300,320$ \\
$\mathrm{Mb} / \mathrm{H}_{2} \mathrm{O}:$ :gly $50: 50$ wt $\%$ & $h=1$ & 304 & \\
$\mathrm{Mb} / \mathrm{D}_{2} \mathrm{O}:$ d-gly $50: 50$ wt $\%$ & & 278 & \\
\hline \hline
\end{tabular}

$\pm 0.5 \mathrm{meV}$ were obtained. For all measurements the samples were placed in flat $\mathrm{Al}$ containers with an internal thickness (i.e., sample thickness) of about $0.3 \mathrm{~mm}$, and the data were collected with the sample holder oriented under an angle of $140^{\circ}$ relative to the transmitted neutron beam. The 51 detectors, each corresponding to a specific scattering angle and therefore also a specific $Q$-value (momentum transfer) at zero energy transfer, were grouped into 11 groups of three detectors per group, giving a total $Q$-range of 0.46-1.52 $\AA^{-1}$.

In our quasielastic neutron scattering (QENS) measurements the measured spectra $I_{m}(Q, \omega)$ are a convolution of the scattering law $S(Q, \omega)$ with the resolution function $R(Q, \omega)$ of the instrument (in our case determined by the scattering from Vanadium $)$, i.e., $I_{m}(Q, \omega)=S(Q, \omega) \otimes R(Q, \omega)$. Вy applying a Fourier transform of the measured data the convolution is reduced to the multiplication $I_{m}(Q, t)=I(Q, t)$ $\times R(Q, t)$ in time domain (note here the change of notation). The intermediate scattering function $I(Q, t)$ can then easily be obtained by dividing the Fourier transformed data with the Fourier transform of the resolution function $(I(Q, t)$ $\left.=I_{m}(Q, t) / R(Q, t)\right)$. Both the data corrections as well as the actual Fourier transforms were performed using the onsite program modes. ${ }^{38}$ This program applies a discrete complex Fourier integral to the measured spectra

$$
I_{m}(Q, t)=\sum_{k=1}^{N} I_{m}\left(Q, \omega_{k}\right) \exp \left(-i \omega_{k} t\right) \Delta \omega_{k}
$$

In this equation $N$ denotes the number of time-of-flight channels, $\omega_{k}$ is the angular frequency, and $\Delta \omega_{k}$ is the width of channel $k$. For our analysis the data were not subjected to binning or interpolation on the energy scale before the numerical Fourier transform was applied in 100 steps (i.e., in steps of $0.01 \mathrm{meV})$ in the energy range $\Delta E=\hbar \omega$ $= \pm 0.5 \mathrm{meV}$.

In general, the intermediate scattering function $I(Q, t)$ can be described by one or several Kohlrausch-WilliamWatts (KWW) stretched exponential functions. In the case of the scattering from the deuterated samples (mainly caused by the protein hydrogens) one KWW function was used to describe the data. For these samples two different curve fitting approaches were applied. The first one was performed with the assumption that $I(Q, t)$ decays to zero at long times [first part of Eq. (2), i.e., $A=B=0]$. Thus, with this approach, it is likely that we obtain the shortest possible relaxation times averaged over all protein relaxations (also on longer time scales than here measured). This approach gives rise to a very strong, and, at low $Q$-values, an unrealistic $Q$-dependence of the protein relaxation time. To overcome this problem and the severe extrapolation of $I(Q, t)$ to longer times, a second approach to fit the $I(Q, t)$ data was also used. With this approach we tried to fit the data for all $Q$-values using fixed values of both the protein relaxation time $\tau_{p}$ and the stretching parameter $\beta_{\mathrm{KWw}}$. The value of $\beta_{\mathrm{KWW}}(=0.51)$ was even assumed to be the same for all temperatures and all samples. Thus, we assumed that the protein relaxations observed on the experimental time scale were of rotational or other local character, and therefore exhibit a $Q$-independent average relaxation time, but $Q$-dependent relaxation amplitude. Hence, parameter $B$ in Eq. (2) was a free fit parameter for approach 2, whereas parameter $A=0$. Based on previous studies (see, e.g., Ref. 32) this assumption should be reasonable at least for low $Q$-values since no long range protein motions are expected on the short experimental time scale. However, at the largest $Q$-values this approach might be misleading and approach 1 is likely to better estimate the $Q$-dependence of the protein dynamics.

For the scattering from the protonated samples (containing information about the dynamics of both the protein and the surrounding solvent) a sum of two KWW functions was used, where one describes the protein and the other one describes the solvent dynamics, respectively. Thus, for fits to the protonated samples we used the following equation:

$$
\begin{aligned}
I(Q, t)= & \left.(1-A-B) \exp \mid-\left(\frac{t}{\tau_{p}}\right)^{\left\langle\beta_{\mathrm{KWW}_{p}}\right\rangle}\right] \\
& +A \exp \left[-\left(\frac{t}{\tau_{s}}\right)^{\beta_{\mathrm{KWw}_{s}}}\right]+B \quad\left(0<\beta_{\mathrm{KWW}}<1\right),
\end{aligned}
$$

where $\tau_{p}$ and $\left\langle\beta_{\mathrm{KWw}}\right\rangle$ are the typical relaxation time and the average stretching parameter related to the protein dynamics, and $\tau_{s}$ and $\beta_{\mathrm{KWW} s}$ are the corresponding parameters for the solvent dynamics. $A$ denotes the relative amplitude of the solvent relaxation, which means that the relative amplitude 
of the protein relaxation is given by $1-A$ since $I(Q, t=0)$ is normalized to $1 . B$ is the $Q$-dependent constant used in the second fitting approach. The parameters describing the protein dynamics in the protonated samples were kept fixed to the values obtained from fits to the deuterated samples. The assumption that the protein dynamics is unaffected by the deuteration of the solvent is not fully correct due to the known stabilizing effect of deuterium and the slightly lower number of solvent molecules for a fixed weight fraction. However, the error in this assumption should be small compared to other sources of error. Furthermore, the fact that good fits to the data are obtained with this assumption and that the results obtained for the solvent dynamics are almost independent on the approach used to describe the protein dynamics supports its validity. Thus, the fitting parameters obtained from both approaches 1 and 2 can be used to "subtract" the protein dynamics on the experimental time scale properly so that the $Q$-dependence of the solvent dynamics is correctly extracted.

\section{RESULTS}

Some typical QENS spectra taken at $300 \mathrm{~K}$ and $Q$ $=1.06 \AA^{-1}$ for myoglobin in water and a water:glycerol mixture of 50:50 wt \% are shown in Fig. 1 together with the resolution function given by the scattering from vanadium. As shown in this figure the quasielastic broadening of the elastic peak is larger for the protonated and deuterated myoglobin samples containing only water as a solvent compared to the samples containing a water:glycerol mixture at the same total solvent level $(h=1)$.

In Fig. 2 the intermediate scattering functions $I(Q, t)$ for the deuterated samples curve fitted by approach 1 are shown. From the figure it is clear that in the accessible $Q$-range, the measured $I(Q, t)$ for all samples are well described by one KWW function [Eq. (2) with $A=B=0$ ]. By use of the characteristic relaxation times and the stretching parameters from these fits, average relaxation times were calculated according to the relation

$$
\left\langle\tau_{p}\right\rangle=\frac{\tau_{p}}{\beta_{\mathrm{KWw}_{p}}} \Gamma\left(\frac{1}{\beta_{\mathrm{KWw}_{p}}}\right),
$$

where $\Gamma$ is the gamma function, and $\beta_{\mathrm{Kww} p}$ is the average stretching parameter obtained from Eq. (2). The average relaxation times $\left(\left\langle\tau_{p}\right\rangle\right)$ are given in Table II, and the stretching parameters $\left(\beta_{\mathrm{Kww} p}\right)$ in Table III. In order to study the nature of the dynamics in more detail, the average relaxation rates $\left(1 /\left\langle\tau_{p}\right\rangle\right)$ obtained for the deuterated samples are plotted as a function of $Q^{2}$ in Fig. 3. From this figure it is seen that the $Q$-dependence of $1 /\left\langle\tau_{p}\right\rangle$ is well described by a power law $a Q^{b}$, with $b=2.5 \pm 0.3$ for all samples except for myoglobin in $\mathrm{D}_{2} \mathrm{O}$ at $260 \mathrm{~K}$, where the value of parameter $b$ appears to be somewhat lower. In this case, where the sample contains a small amount of ice, $b=1.9 \pm 0.1$.

The curve fitted intermediate scattering functions $I(Q, t)$ by approach 2 are shown in Fig. 4 for some of the deuterated
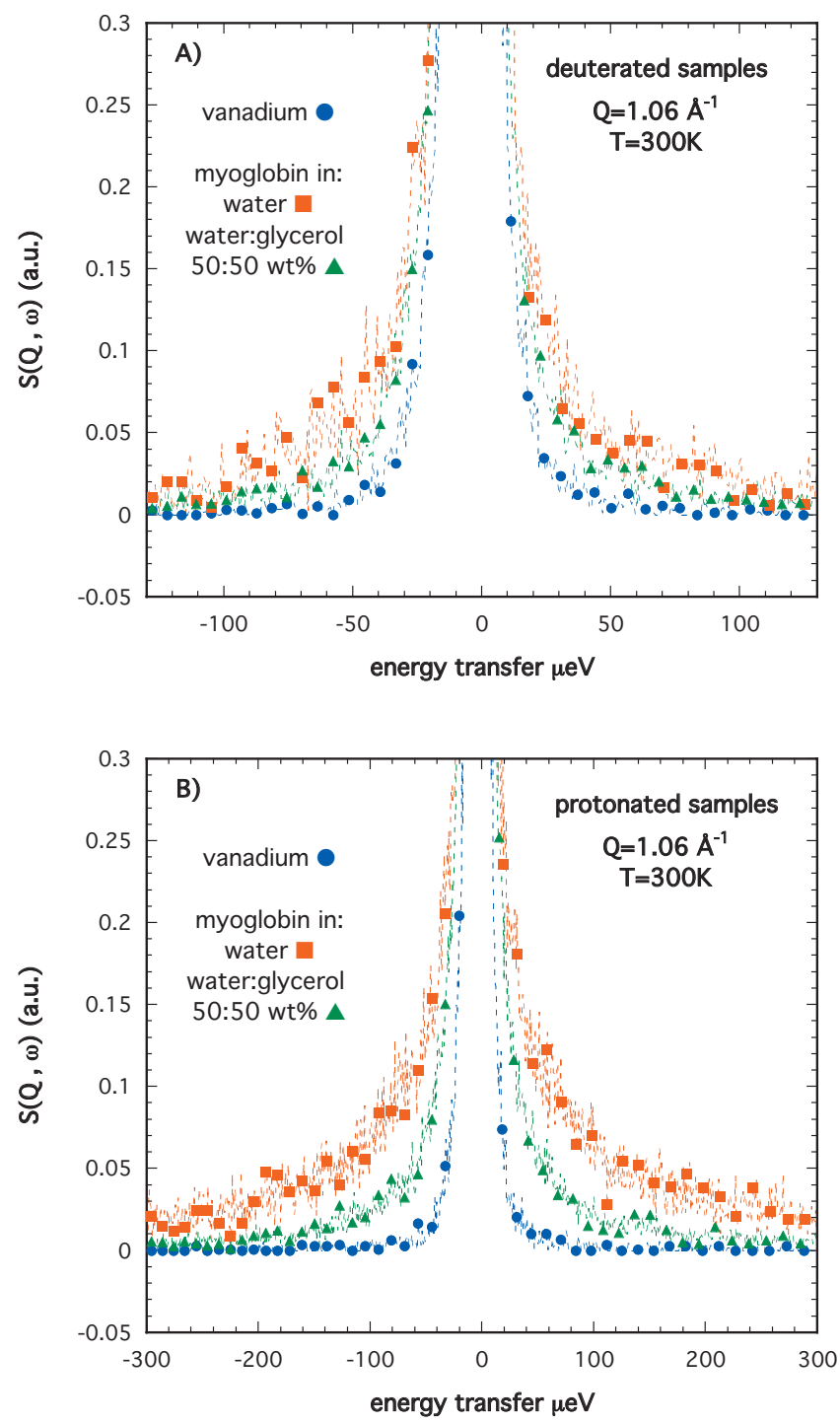

FIG. 1. (Color) QENS spectra at $300 \mathrm{~K}$ for myoglobin in water at the hydration level $h=1$, and in a mixture of 50:50 wt \% of water and glycerol at the solvent level $h=1$. The resolution $(\mathrm{FWHM}=17.5 \mu \mathrm{eV})$ of the spectrometer is given by the scattering from vanadium. For comparison all spectra are normalized to 1 at zero energy transfer.

samples, together with the obtained $Q$-dependent amplitude of relaxation. The average relaxation times $\left(\left\langle\tau_{p}\right\rangle\right)$ are given in Table II. From this analysis it is clear that also with this approach it is possible to describe the data reasonably well.

As described above in the experimental section, in order to determine the contribution of the solvent to the scattering from the protonated samples the parameters describing the protein dynamics (i.e., $\tau_{p}$ and the $\beta_{\mathrm{KWW} p}$ ) were fixed in Eq. (1) to the values obtained from fits according to approach 1 to the deuterated samples. In Fig. 5 the intermediate scattering function $I(Q, t)$ is shown for these samples, and in Table III the stretching parameters $\beta_{\mathrm{KWWs}}$ of the solvents are given. In Fig. 6 the average relaxation rates $1 /\left\langle\tau_{s}\right\rangle$ obtained for the solvent dynamics in these samples are plotted as a function of $Q^{2}$. As common for both bulk and confined water, the data in Fig. 6 are fitted by the Gaussian jump-length diffusion model 

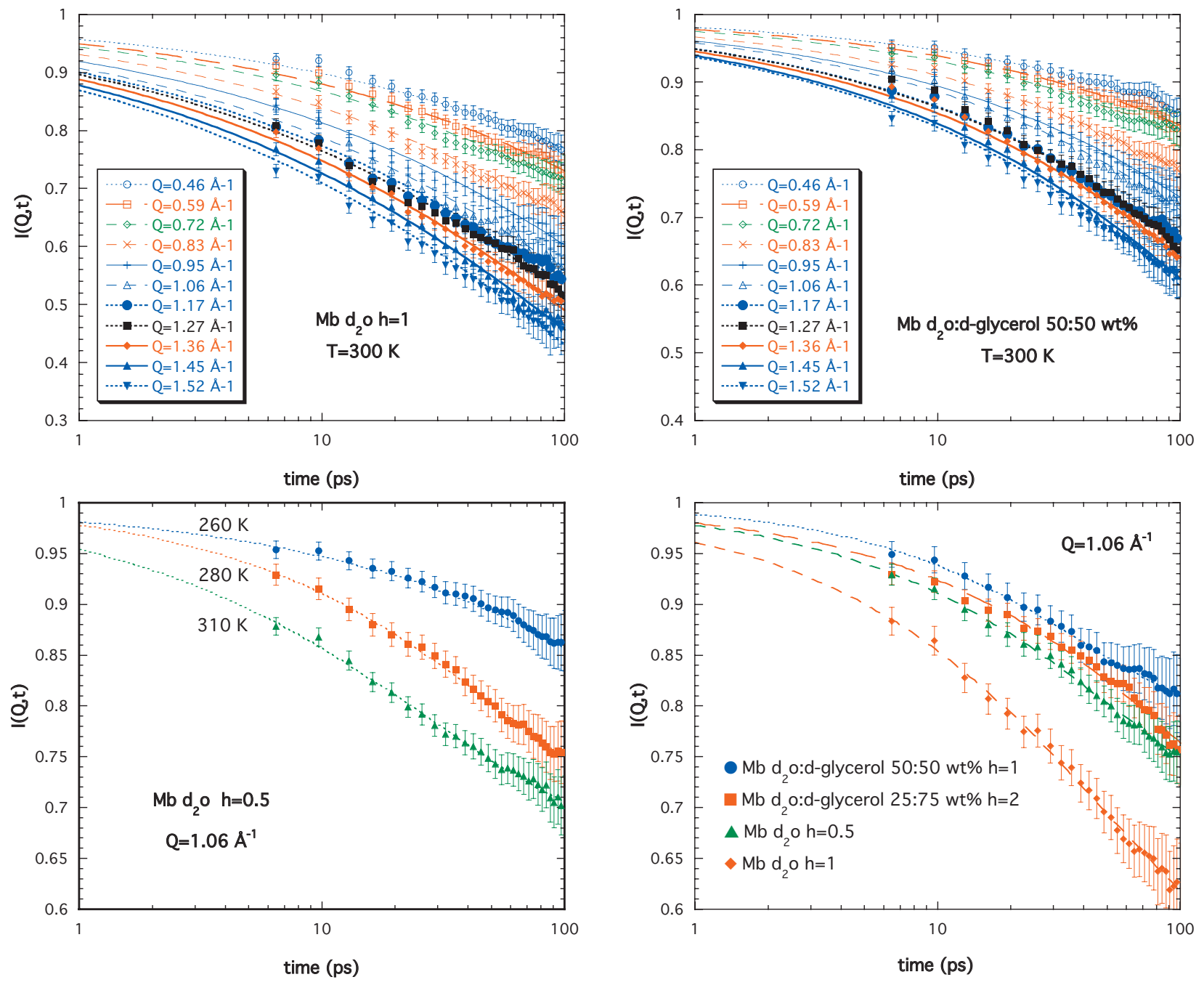

FIG. 2. (Color) Intermediate scattering functions $I(Q, t)$ for different $Q$-values, temperatures, and samples as given in the plots. The temperatures in the lower panel to the right are $280 \mathrm{~K}$ for all samples except for myoglobin in $\mathrm{D}_{2} \mathrm{O}$ :d-glycerol $25: 75 \mathrm{wt} \% h=2$, where the $T$ is $290 \mathrm{~K}$. The lines are fits to the KWW function used for the fitting procedure by approach 1 . The values of the stretching parameter $\left\langle\beta_{\mathrm{KWw}}\right\rangle$ are given in Table III.

$$
\frac{1}{\langle\tau\rangle}=\frac{1}{\tau_{\text {res }}}\left[1-\exp \left(-\frac{Q^{2}\left\langle r^{2}\right\rangle}{6}\right)\right],
$$

where $\left\langle r^{2}\right\rangle$ is the MSD and $\tau_{\text {res }}$ is the average residence time between two jumps, respectively. By use of the parameters derived from the fits to Eq. (4) a diffusion constant $D_{s}$ can be determined according to

$$
D_{s}=\left\langle r^{2}\right\rangle / 6 \tau_{\text {res }} .
$$

Values for $D_{s},\left\langle r^{2}\right\rangle$, and $\tau_{\text {res }}$ are given in Table IV. In addition, the diffusion constants are shown graphically in Fig. 7.

\section{DISCUSSION}

From Table IV and Fig. 7 it is obvious that the diffusion constants $\left(D_{s}\right)$ obtained for the solvents are different from

TABLE II. Relaxation times obtained for the protein as obtained by approach 1 and 2 at $Q \approx 1 \AA^{-1}$.

\begin{tabular}{lccc}
\hline \hline Sample & $\begin{array}{c}\text { Temperature } \\
(\mathrm{K})\end{array}$ & $\begin{array}{c}\left\langle\tau_{p}\right\rangle\left(10^{-9} \mathrm{~s}\right) \\
\text { approach } 1\end{array}$ & $\begin{array}{c}\left\langle\tau_{p}\right\rangle\left(10^{-10} \mathrm{~s}\right) \\
\text { approach } 2\end{array}$ \\
\hline $\mathrm{Mb} / \mathrm{D}_{2} \mathrm{O} h=0.5$ & 280 & $3.7 \pm 0.4$ & $4.4 \pm 0.4$ \\
& 310 & $2.4 \pm 0.2$ & $2.3 \pm 0.2$ \\
$\mathrm{Mb} / \mathrm{D}_{2} \mathrm{O} h=1$ & 280 & $1.7 \pm 0.2$ & $1.4 \pm 0.1$ \\
& 300 & $1.4 \pm 0.1$ & $0.8 \pm 0.1$ \\
$\mathrm{Mb} / \mathrm{D}_{2} \mathrm{O}:$ d-gly $25: 75 \mathrm{wt} \% h=2$ & 320 & $1.2 \pm 0.1$ & $0.6 \pm 0.1$ \\
$\mathrm{Mb} / \mathrm{D}_{2} \mathrm{O}:$ d-gly $50: 50 \mathrm{wt} \% h=1$ & 290 & $3.8 \pm 0.4$ & $6.0 \pm 0.6$ \\
& 280 & $8.8 \pm 0.9$ & $9.1 \pm 0.9$ \\
& 300 & $2.7 \pm 0.3$ & $3.5 \pm 0.3$ \\
& 320 & $2.1 \pm 0.2$ & $1.6 \pm 0.2$ \\
\hline \hline
\end{tabular}


TABLE III. The values of the shape parameter $\left\langle\beta_{\mathrm{KWW}}\right\rangle$ and $\left\langle\beta_{\mathrm{KWW}}\right\rangle$ obtained for the protein and the solvents, respectively, averaged over all $Q$ values, for all samples and temperatures. The estimated error is for all samples and temperatures less then $10 \%$.

\begin{tabular}{lccc}
\hline \hline Sample & Temperature $(\mathrm{K})$ & $\left\langle\beta_{\mathrm{Kww}}\right\rangle$ & $\left\langle\beta_{\mathrm{Kww}}\right\rangle$ \\
\hline $\mathrm{Mb} / \mathrm{H}_{2} \mathrm{O} h=0.5$ & $260,280,310$ & & $0.91,0.81,0.80$ \\
$\mathrm{Mb} / \mathrm{D}_{2} \mathrm{O} h=0.5$ & & $0.45,0.45,0.38$ & \\
$\mathrm{Mb} / \mathrm{H}_{2} \mathrm{O} h=1$ & $280,300,320$ & & $0.80,0.76,0.75$ \\
$\mathrm{Mb} / \mathrm{D}_{2} \mathrm{O} h=1$ & & $0.42,0.39,0.39$ & 0.68 \\
$\mathrm{Mb} / \mathrm{H}_{2} \mathrm{O}:$ gly $25: 75$ wt $\% h=2$ & 290 & & 0.47 \\
$\mathrm{Mb} / \mathrm{D}_{2} \mathrm{O}:$ d-gly $25: 75$ wt $\% h=2$ & & & $0.76,0.83,0.74,0.73$ \\
$\mathrm{Mb} / \mathrm{H}_{2} \mathrm{O}:$ gly $50: 50$ wt $\% h=1$ & $260,280,300,320$ & $0.54,0.44,0.45,0.41$ & \\
$\mathrm{Mb} / \mathrm{D}_{2} \mathrm{O}:$ d-gly $50: 50$ wt $\% h=1$ & & & \\
\hline \hline
\end{tabular}

those of the unmixed solvents (pure water and pure glycerol), and that $D_{s}$ decreases with increasing glycerol content and decreasing temperature. In the case of only water $\left(\mathrm{H}_{2} \mathrm{O}\right)$ as a solvent it is evident that the diffusion constant $D_{s}$ of the water increases slightly with increasing hydration level since at $300 \mathrm{~K}$ the water diffusion is a factor $2.0 \pm 0.3$ and $2.6 \pm 0.3$ slower than bulk water for the sample at hydration levels of $h=1$ and $h=0.5$, respectively. The reason for this is, of course, that a larger fraction of the water molecules interacts with the protein surface, and thereby slows down, at the lower hydration level.

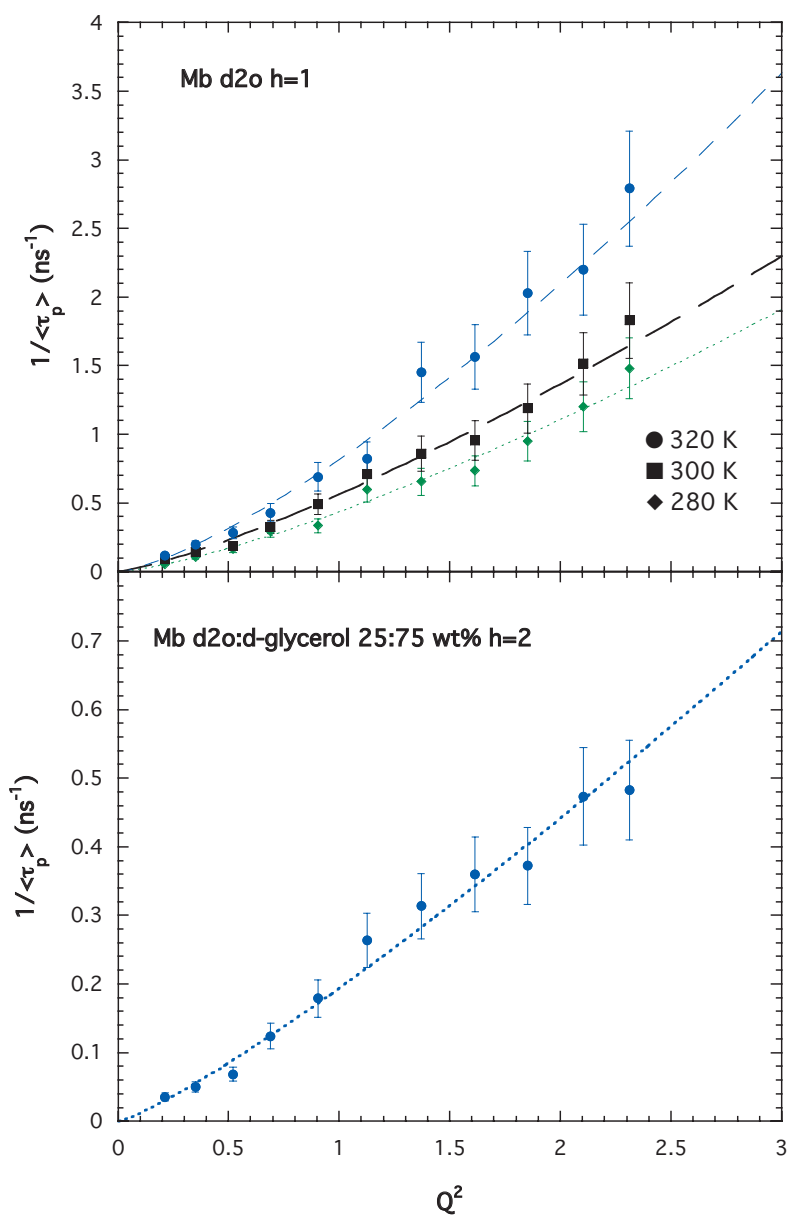

The relative small effect on the average diffusion constant, as well as average relaxation times, for the hydration water of proteins as compared to bulk water is well established by experimental studies using different techniques, such as neutron scattering, 9,39,40 various NMR techniques, ${ }^{41,42}$ and by MD simulations. ${ }^{43-45}$ A similar slowing down of the dynamics is, furthermore, not only typical for protein hydration water but also for hydration water in other biological systems, such as lipid membranes ${ }^{46}$ and carbohydrates, ${ }^{47}$ but in sharp contrast to water confined in many hydrophilic model systems and geological systems,

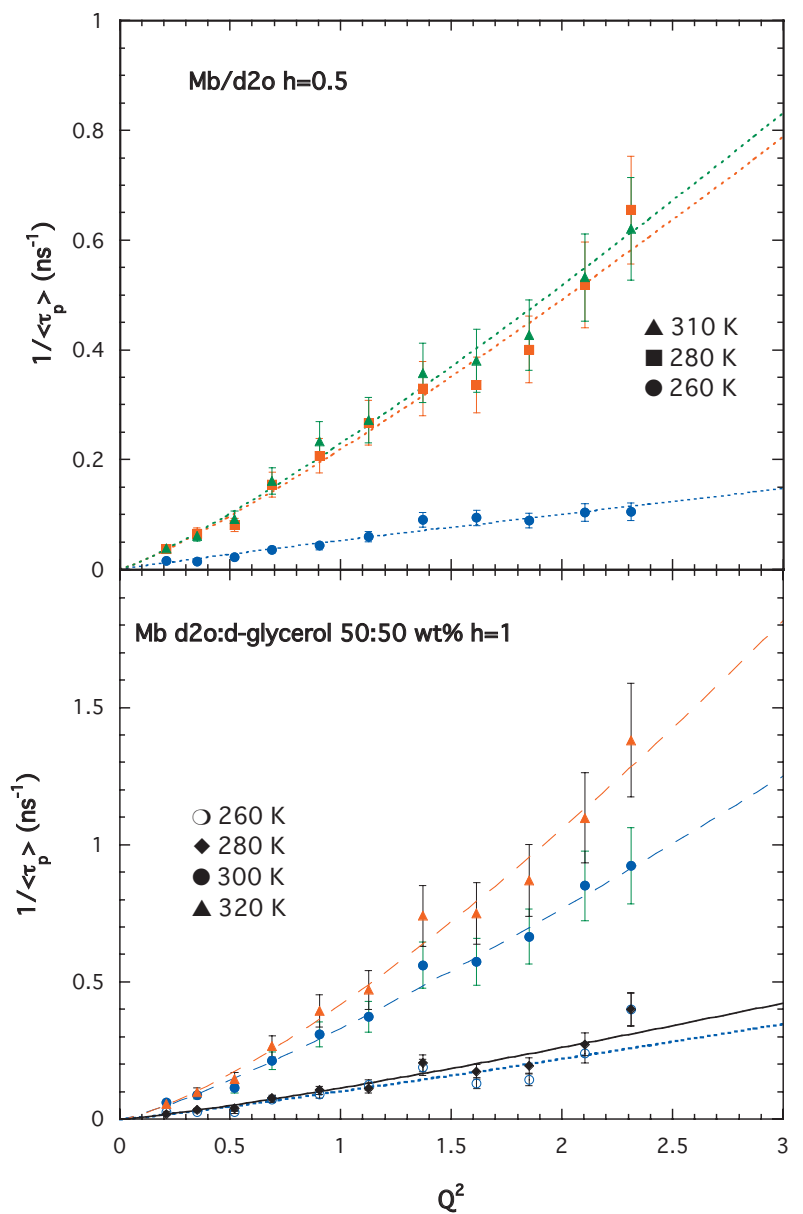

FIG. 3. (Color) The reciprocal average relaxation time $\left(1 /\left\langle\tau_{p}\right\rangle\right)$ obtained by approach 1 for the deuterated samples as a function of $Q^{2}$ for all temperatures. The lines are fits to a power law $a Q^{b}$, where $b$ is around 2.5 for all samples except for myoglobin in $\mathrm{D}_{2} \mathrm{O} h=0.5$ at $260 \mathrm{~K}$ (small amount of ice), where $b$ seems to be somewhat lower. 

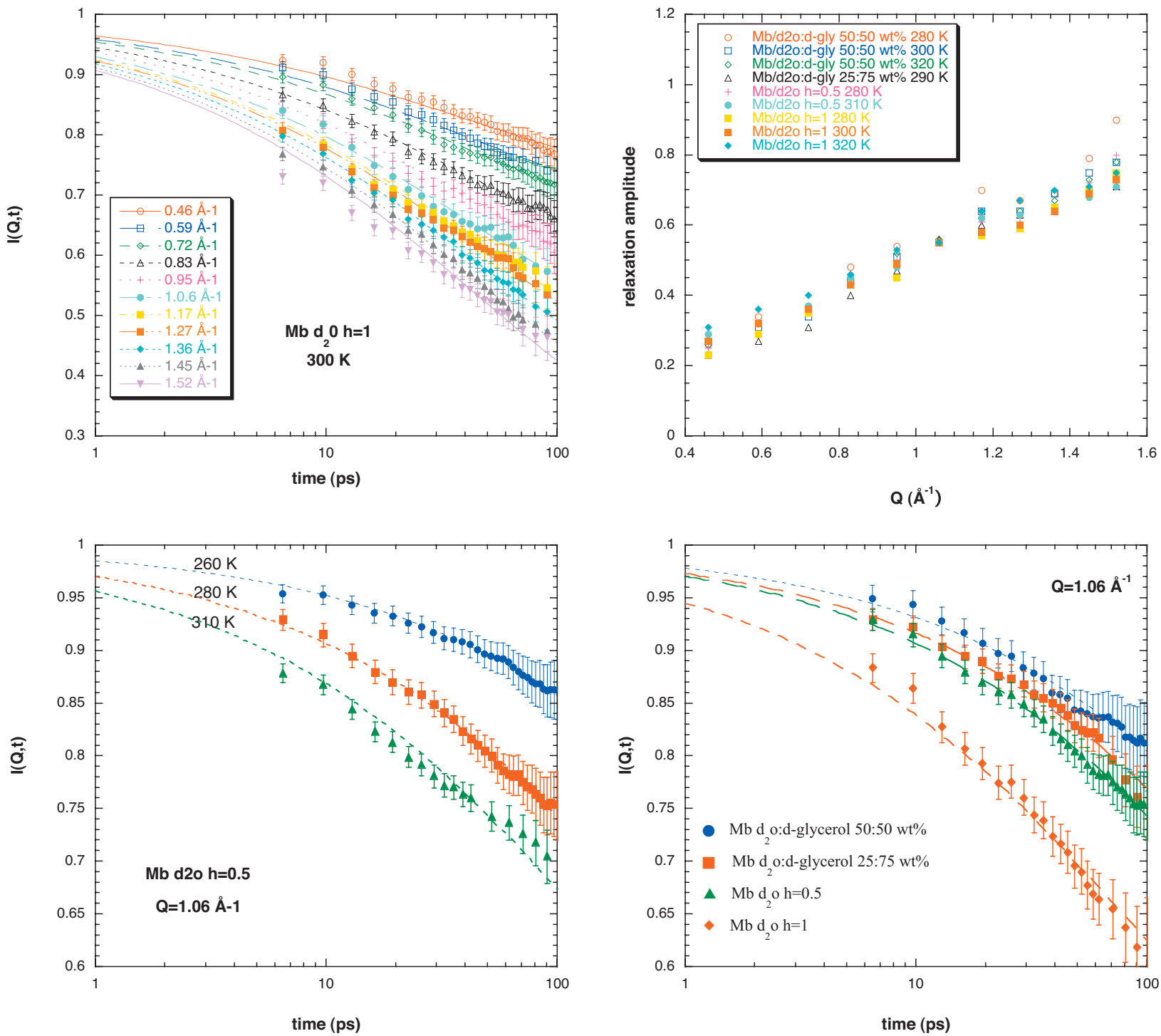

FIG. 4. (Color) Intermediate scattering function $I(Q, t)$ for different $Q$-values and temperatures together with the amplitudes of the relaxation obtained from the curve fitting made by approach 2 . The stretching parameter $\beta_{\mathrm{KWw}}$ are for all samples $\approx 0.51$ and the decay of the relaxation was determined to a level of 0.45 at $Q=1.06 \AA^{-1}$ for all samples. The obtained relaxation times for each sample are given in Table II.

such as a molecular sieve of pore size $10 \AA$ (Ref. 48) and a fully hydrated clay, ${ }^{49}$ where the diffusion constant is reduced by as much as a factor 30 or more at room temperature. Thus, despite that the biological systems and these other systems all are of hydrophilic nature the water dynamics is considerably less affected compared to bulk water in the biological systems. A possible explanation for such behavior can be that the biomolecular surfaces are less hydrophilic/polar than the surfaces inside the pores of the other systems, and consequently, the interaction between the water molecules and the surface is weaker in case of biomolecular surfaces. This is further supported by the fact that the protein surface is not entirely hydrophilic, but also contains slightly hydrophobic parts. $^{50}$

For the solvent in the $\mathrm{Mb} / \mathrm{H}_{2} \mathrm{O}$ : glycerol 50:50 wt \% sample at $300 \mathrm{~K} D_{s}$ is reduced by a factor $3.0 \pm 0.8$ and $3.6 \pm 0.6$ compared to the samples containing only water as a solvent having the same water content $(h=0.5)$ and the same total solvent level $(h=1)$, respectively. By changing the ratio of water to glycerol to $25: 75 \mathrm{wt} \%$ and increasing the total solvent level to $h=2$ (twice the 50:50 wt \% sample) the diffusion of the solvent molecules surrounding the protein is about $3.6 \pm 1.3$ and $11 \pm 3$ times slower compared to $D_{s}$ of the solvent in the 50:50 wt $\% \mathrm{H}_{2} \mathrm{O}$ : glycerol sample, and the sample containing the same amount of water, i.e., $\mathrm{Mb} / \mathrm{H}_{2} \mathrm{O}$ $h=0.5$, respectively. Not surprisingly, the addition of glycerol to the solvent results in a decreasing root mean square jump length $\left\langle r^{2}\right\rangle^{1 / 2}$, and an increasing residence time $\tau_{\text {res }}$ (see Table IV), which together give rise to the reduced diffusion with increasing glycerol content.

However, the main question addressed in this study is how different solvents and solvent compositions influence the dynamics of proteins. As described in the experimental section, two different approaches with different assumptions were used to describe the measured data. With approach 1 the idea is that the intermediate scattering function $I(Q, t)$ decays to zero at longer times whereas approach 2 is based on the assumption that the protein relaxation can be described by $Q$-independent values of the stretching parameter 

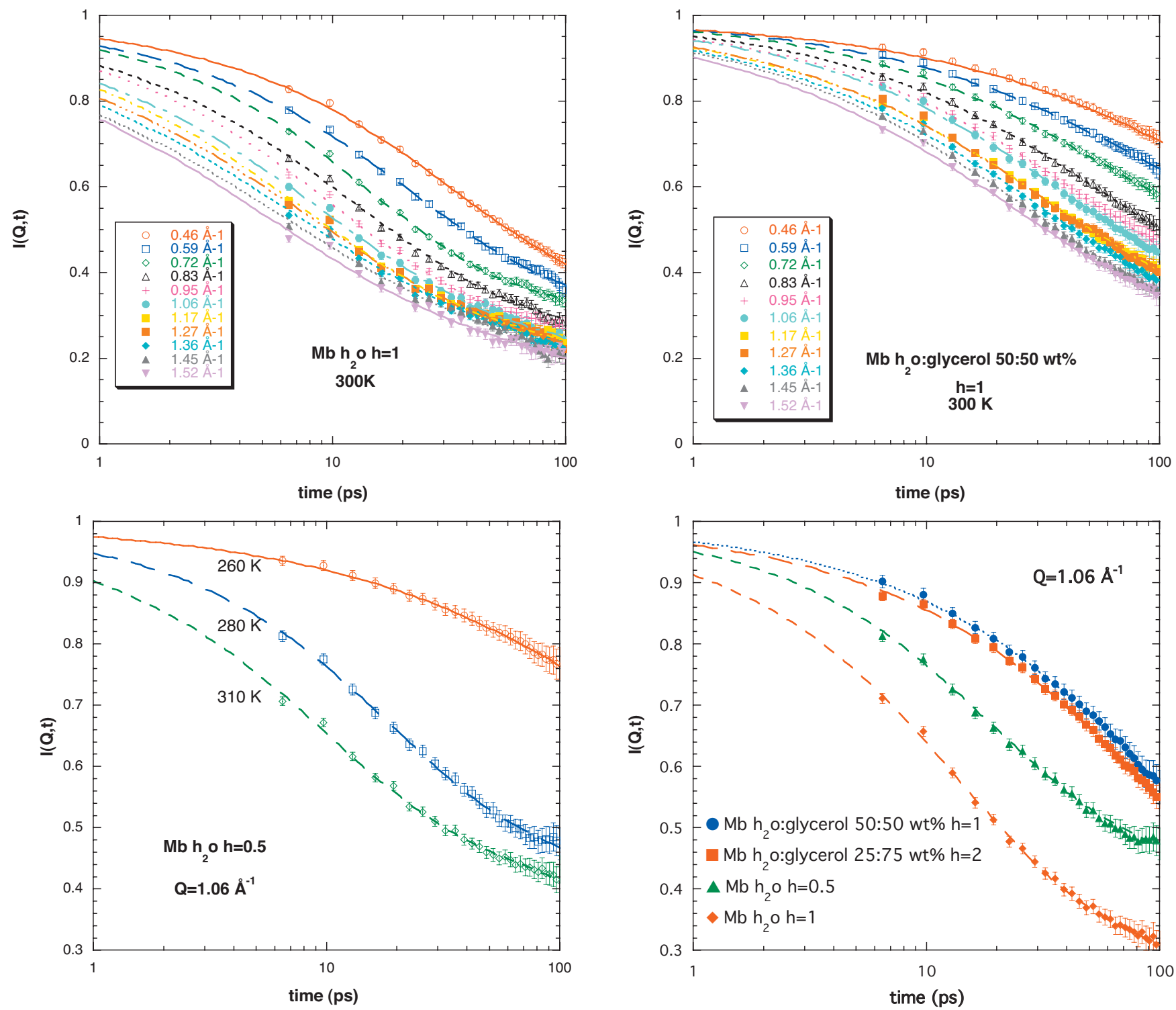

FIG. 5. (Color) Intermediate scattering functions $I(Q, t)$ obtained for the protonated samples for different $Q$-values and temperatures. The temperatures in the lower panel to the right are $280 \mathrm{~K}$ for all samples except for myoglobin in $\mathrm{H}_{2} \mathrm{O}$ : glycerol $25: 75 \mathrm{wt} \% h=2$, where the $T$ is $290 \mathrm{~K}$. The experimental data were fitted to a sum of two KWW functions [Eq. (2)], one showing the protein dynamics and the other showing the solvent dynamics, respectively.

$\beta_{\mathrm{KWw}}$ and the average relaxation time $\left\langle\tau_{p}\right\rangle$. As expected, the two different approaches give very different average relaxation times, where, e.g., at $T=280 \mathrm{~K}$ and $Q \approx 1 \AA^{-1}\left\langle\tau_{p}\right\rangle$ obtained by approach 1 is about a factor 10 slower compared to $\left\langle\tau_{p}\right\rangle$ obtained by approach 2 for all samples (see Fig. 8). However, it is important to note that independent of the chosen approach, it is clear that the $Q$-dependence of the protein dynamics must be very different to that of the corresponding solvents since a Gaussian jump-length diffusion model can certainly not be used to describe the $Q$-dependence of the protein dynamics. Furthermore, within each approach, it seems that the nature of the determined protein relaxations are of the same type and therefore likely to be comparable for the protein in all solvents because with approach 1 the power law exponent $b$ becomes the same for all ice-free samples, and both approaches work approximately equally well for all samples. Thus, even if the results are of different character for the different approaches, they imply that the dynamical behavior of the protein is similar in all solvent compositions. This is further supported by the fact that both the structure and the specific activity of proteins persists in almost anhydrous glycerol ${ }^{51}$ even if the activity most likely is somewhat reduced compared to when it is in its natural environment. ${ }^{51}$

Before we discuss the relation between protein and solvent dynamics we have to see how both the average protein relaxation time as well as the diffusion constant of the solvent depend on the solvent composition. Let us first look at the values presented in Table II for approach 1 (approach 2 gives slightly different numerical values, but qualitatively the same results). In the case of pure water as solvent the average relaxation time of the protein $\left\langle\tau_{p}\right\rangle$ increases substantially with decreasing hydration level. At $T=280 \mathrm{~K}$ and $Q$ $\approx 1.0 \AA^{-1}\left\langle\tau_{p}\right\rangle$ is about a factor $2.2 \pm 0.5$ faster for the higher $(h=1.0)$ compared to the lower $(h=0.5)$ hydration level. This decrease in the protein relaxation time with in- 

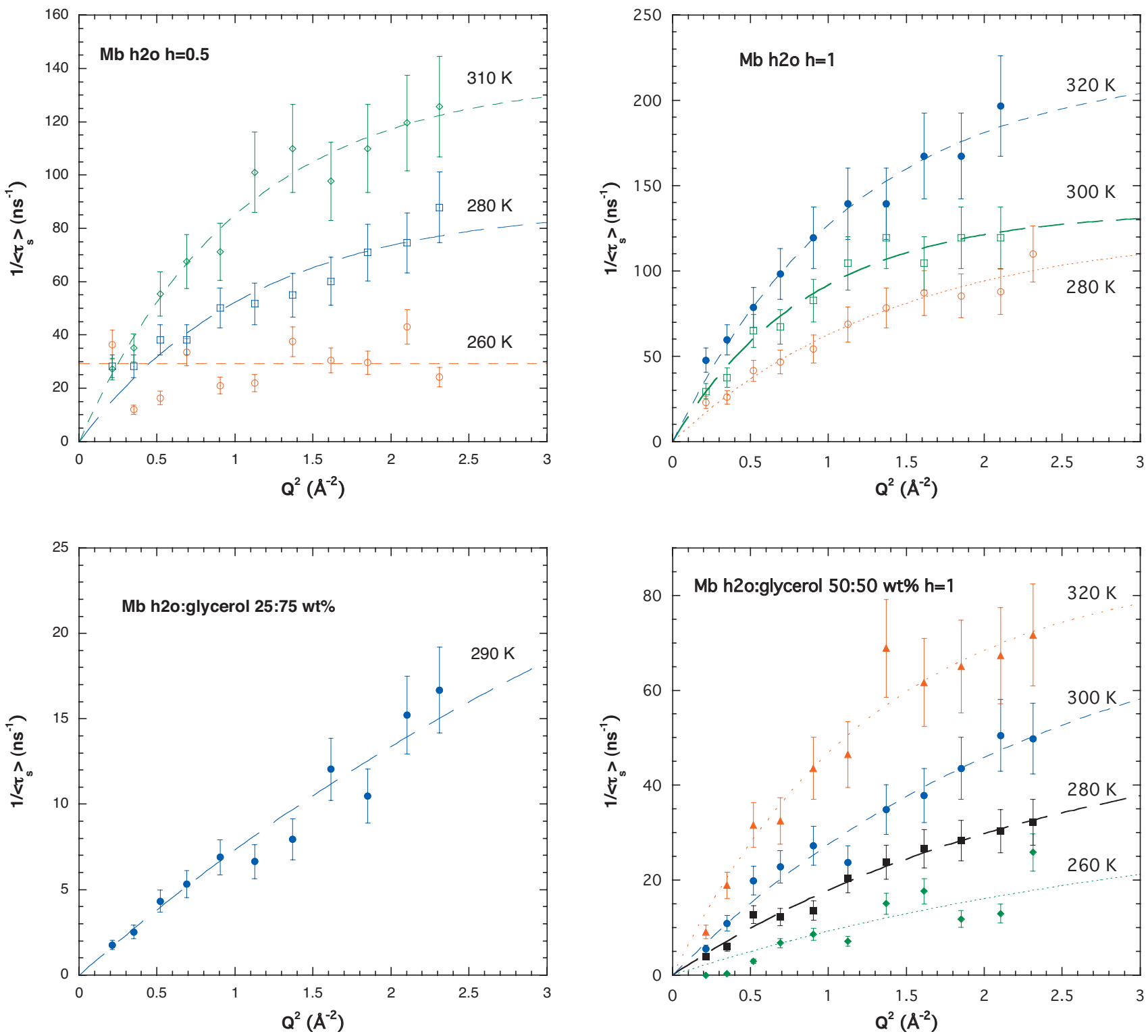

FIG. 6. (Color) The reciprocal average relaxation time $\left(1 /\left\langle\tau_{s}\right\rangle\right)$ obtained for the solvent dynamics as a function of $Q^{2}$ for all temperatures. The lines show the $Q^{2}$-dependence for $1 /\left\langle\tau_{s}\right\rangle$ as described by the Gaussian jump diffusion model [Eq. (4)].

creasing hydration level can be compared with the corresponding increase in $D_{s}$ for the solvent, which is only a factor $1.1 \pm 0.3$ at $T=280 \mathrm{~K}$ (see Table IV). For the protein in the 50:50 wt \% water:glycerol solvent the average relaxation time at $T=280 \mathrm{~K}$ and $Q \approx 1.0 \AA^{-1}$ is reduced by a factor $2.4 \pm 0.6$ and $5.2 \pm 1.0$ compared to the protein at the hydration level $h=0.5$ (that contains the same amount of water), and at the higher hydration level $h=1.0$ (that contains the same amount of solvent), respectively. The corresponding decrease in $D_{s}$ for the solvent are given by the factors $3.4 \pm 0.9$ and 3.9 \pm 1.0 , respectively. By increasing the glycerol content to $25: 75 \mathrm{wt} \%$ water:glycerol and the total solvent content to $h=2$ the protein average relaxation time $\left\langle\tau_{p}\right\rangle$ at $290 \mathrm{~K}$ is about the same (a factor $1.0 \pm 0.3$ slower) as for $\mathrm{Mb} / \mathrm{D}_{2} \mathrm{O} h=0.5$ at $280 \mathrm{~K}$, and becomes a factor of $2.3 \pm 0.5$ slower (at the same temperature) compared to the protein hydrated to $h=1.0$. This relatively small slowing down of the protein dynamics in the $\mathrm{Mb} / \mathrm{D}_{2} \mathrm{O}$ : glycerol 25:75 wt \% sample is in contrast to the decrease in the corresponding $D_{s}$ by a factor of 10 or more compared to the hydration water in the samples $\mathrm{Mb} / \mathrm{D}_{2} \mathrm{O} h=0.5$ and $h=1.0$. Here it should be noted that for the sample with 25:75 wt \% water:glycerol, we cannot exclude a significant contribution from coherent solvent scattering to $\left\langle\tau_{p}\right\rangle$. Thus, in this case the average relaxation time $\left\langle\tau_{p}\right\rangle$ is averaged over both the protein dynamics and the coherent contribution from the faster solvent dynamics, which tends to make $\left\langle\tau_{p}\right\rangle$ somewhat lower than its real value for only the protein. Another possible explanation for the disagreement between the magnitude of the slowing down of protein dynamics compared to the slowing down of the corresponding $D_{s}$ is that the environment closest to the protein molecule most likely is different to that of the surrounding bulk solvent. According to a recent small angle neutron scattering study on the globular protein lysozyme ${ }^{52}$ glycerol is preferentially excluded from the protein surface when water is present in the solvent. In that study the structure of the protein and its surrounding was investigated as a 
TABLE IV. Parameters that describe the solvent diffusion as determined from the fits to the Gaussian jumplength diffusion model, where $\left\langle r^{2}\right\rangle^{1 / 2}$ is the root MSD, $\tau_{\text {res }}$ is the average residence time between two jumps in the translational jump diffusion process, and $D_{s}$ is the solvent diffusion constant, respectively.

\begin{tabular}{lcccc}
\hline \hline & $\begin{array}{c}\text { Temperature } \\
\text { Prov }\end{array}$ & $\begin{array}{c}\left\langle r^{2}\right\rangle^{1 / 2} \\
(\AA)\end{array}$ & $\begin{array}{c}\tau_{\text {res }} \\
(\mathrm{ps})\end{array}$ & $\begin{array}{c}D_{s} \\
\left(10^{-10} \mathrm{~m}^{2} / \mathrm{s}\right)\end{array}$ \\
\hline $\mathrm{Mb} / \mathrm{H}_{2} \mathrm{O} h=0.5$ & 260 & $\cdots$ & $\cdots$ & $\ldots$ \\
& 280 & $2.15 \pm 0.26$ & $10.40 \pm 1.1$ & $7.4 \pm 0.7$ \\
& 310 & $2.26 \pm 0.20$ & $6.77 \pm 0.7$ & $12.5 \pm 1.3$ \\
$\mathrm{Mb} / \mathrm{H}_{2} \mathrm{O} h=1$ & 260 & $\cdots$ & $\cdots$ & $\cdots$ \\
& 280 & $1.96 \pm 0.20$ & $7.57 \pm 0.8$ & $8.5 \pm 0.9$ \\
& 300 & $2.12 \pm 0.26$ & $5.83 \pm 0.9$ & $12.8 \pm 1.9$ \\
$\mathrm{Mb} \mathrm{H}_{2} \mathrm{O} /$ gly $25: 75 \mathrm{wt} \% h=2$ & 320 & $2.27 \pm 0.20$ & $4.52 \pm 0.6$ & $19.0 \pm 1$ \\
$\mathrm{Mb} \mathrm{H}_{2} \mathrm{O} /$ gly $50: 50 \mathrm{wt} \% \mathrm{~h}=1$ & 290 & $1.08 \pm 0.35$ & $24.76 \pm 4.3$ & $0.78 \pm 0.1$ \\
& 260 & $1.39 \pm 0.65$ & $30.61 \pm 5.6$ & $1.1 \pm 0.2$ \\
& 280 & $1.56 \pm 0.23$ & $18.62 \pm 1.5$ & $2.2 \pm 0.3$ \\
& 300 & $1.75 \pm 0.23$ & $14.31 \pm 1.5$ & $3.6 \pm 0.5$ \\
& 320 & $1.86 \pm 0.24$ & $9.52 \pm 1.2$ & $6.1 \pm 0.9$ \\
\hline \hline
\end{tabular}

function of both different water:glycerol solvent compositions and different deuteration grades and it was, for instance, concluded that besides from that the water is preferentially located at the protein surface the glycerol in the solvent prevents protein-protein contact that, in general, cannot be excluded at low hydration levels. ${ }^{53}$ This means that for high glycerol contents the diffusion constant of the hydration layer may be higher than in the more glycerol rich bulk solvent. Since the protein dynamics is expected to be more sensitive to the dynamics in the hydration layer than in the bulk solvent, which is mainly probed at $h=2$, a relation between $\left\langle\tau_{p}\right\rangle$ and $1 / D_{s}$ may be lost. However, in general our results suggest that the protein relaxation time is not only dependent on the diffusion constant (or in our case the relaxation rate) of the solvent, but also on the total amount of the solvent. For pure water as solvent or a given water:glycerol ratio the solvent relaxation time decreases only slightly with increasing amount of solvent, whereas the protein relaxation time seems to exhibit a much stronger dependence on the amount of solvent.

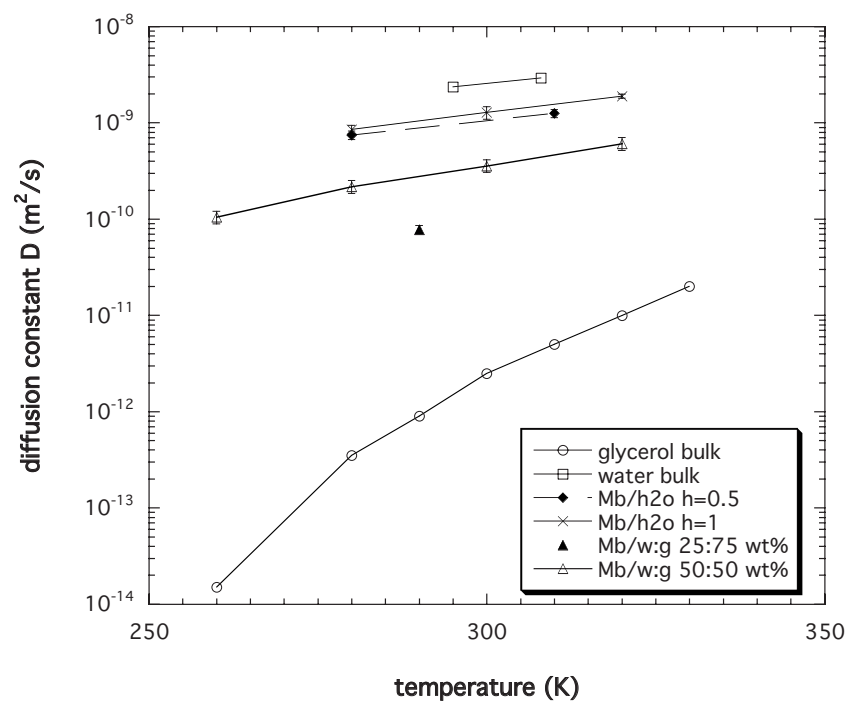

FIG. 7. Diffusion coefficients $\left(D_{s}\right)$ obtained for the investigated samples (also given in Table IV) together with literature values for bulk water (Refs. 64 and 65) and glycerol (Ref. 66). The uncertainties of the data points given in Table IV are about the same size as the symbols.
As mentioned above, the $Q$-dependence of the reciprocal relaxation time $1 /\left\langle\tau_{p}\right\rangle$ obtained by approach 1 is well described by a power law dependence for all samples, where for all temperatures and samples (without ice) the exponent is around $2.5 \pm 0.3$. With approach 2 , on the other hand, we assume a fixed (and sample specific) value of the protein relaxation time $\tau_{p}$ together with a stretching parameter $\beta_{\mathrm{KWW}}$ that was kept at the same value for all samples and temperatures. Thus, in case of approach 2 we assume the protein average relaxation time $\left\langle\tau_{p}\right\rangle$ to be independent of the $Q$-value. Comparatively, most studies ${ }^{32-34}$ found in literature show that the motions of protein hydrogens can be described by confined diffusion, i.e., diffusion inside a sphere, which is displayed by a constant value $\propto 1 /\left\langle\tau_{p}\right\rangle$ at lower $Q$-values, as assumed by approach 2 above. On the other hand, even larger values of the exponent that we obtained with approach 1 have been found in these studies ${ }^{32-34}$ at the highest measured $Q$-values. Thus, approach 1 seems to be able to reproduce a correct $Q$-dependence of the protein dynamics at high $Q$, but fails at low $Q$ where the $Q$-independent dynamics assumed by approach 2 is needed to obtain a realistic $Q$-dependence. Thus, due to the limited experimental time range, where the protein, particularly at low $Q$-values, is far from fully relaxed, it is unfortunately not possible to establish an explicit $Q$-dependence of the protein dynamics over the whole measured $Q$-range, although the results obtained by approaches 1 and 2 most likely display the dynamical behavior at high and low $Q$-values, respectively.

In a neutron scattering experiment the time window is often narrow and limited to a picosecond-nanosecond time scale, which is considerably faster than the time scale of the slowest protein dynamics. It is well known that protein dynamics are not found at a certain time scale since a protein exhibits motions of extremely different characters ranging from very local atomic fluctuations and side chain motions to large scale conformational changes of the entire protein structure. Consequently, protein dynamics involve both different length scales as well as very different time scales, where the time scales are extended over many orders of magnitude. ${ }^{54,55}$ Hence, in order to perform an analysis assumptions have to be made. If the assumptions are not rea- 


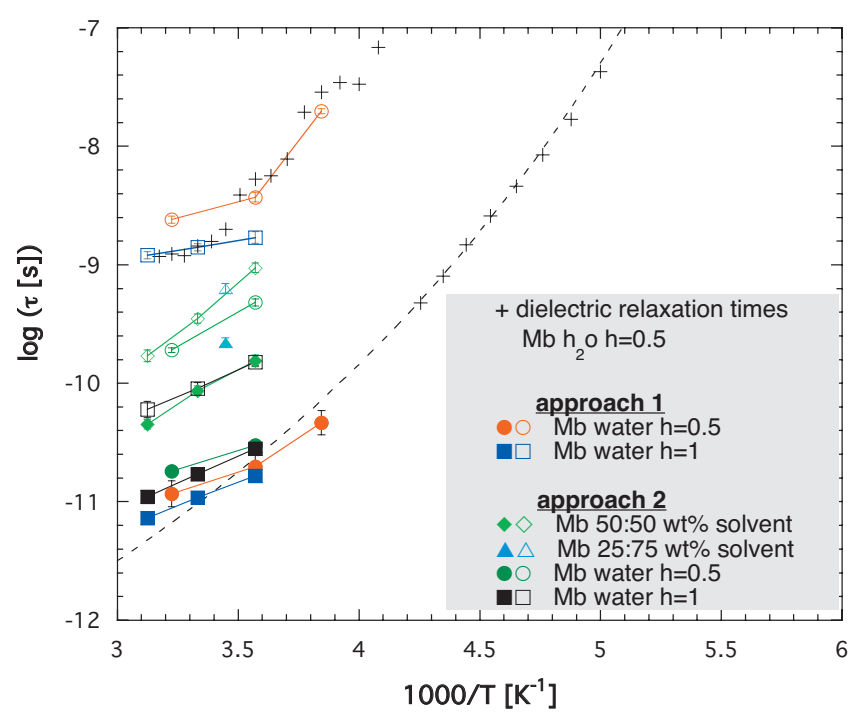

FIG. 8. (Color) Comparison of some of the dielectric relaxation processes obtained for myoglobin in water at the hydration level $h=0.5$ (Ref. 59) with the average relaxation times $\left\langle\tau_{p}\right\rangle_{Q \approx 1 \AA^{-1}}$ (open symbols) and $\left\langle\tau_{s}\right\rangle_{Q \approx 1 \AA^{-1}}$ (filled symbols) for all samples measured in the present study.

sonable, a misleading result, such as an incorrect $Q$-dependence of the dynamics, is likely to be obtained. In contrast to many other studies where the measured dynamic structure factor $S(Q, \omega)$ is analyzed we have, for our samples, chosen to analyze the intermediate scattering function $I(Q, t)$, i.e., the Fourier transform of $S(Q, \omega)$. Advantages of our approach are that it both shows that only a fraction of all protein dynamics is observable in the experimental time window and that there are many different dynamic processes on different time scales, which combined give rise to a very stretched relaxation function. These findings are fully consistent with recent dielectric relaxation data on similar sample compositions, showing several overlapping protein processes. ${ }^{28}$ In fact, from the present experimental data it is clear that even the protein dynamics observed on the experimental time scale involve several processes. Fitting Lorenzian functions to $S(Q, \omega)$ tends, in cases like this, to give relaxation times that are more dependent on the experimental energy resolution.

From this discussion it is evident that $\left\langle\tau_{p}\right\rangle$ depends on how many dynamical processes the average is taken over. With approach 1 the average value is taken over all protein processes, provided that all processes can be described by a single KWW function, whereas with approach 2 we essentially take the average value of the processes observed in the experimental time window. Since the different processes cannot be easily separated in time it is not possible to consider the relaxation times obtained by approach 1 more or less correct than those obtained by approach 2 . Simply speaking, the two approaches are giving answers to somewhat different questions.

The results obtained by approach 1 , which most likely can be applied to higher $Q$-values, might be discussed in terms of heterogeneous and homogenous dynamical scenarios, which can be used to describe the stretched exponential form of $I(Q, t) \cdot{ }^{56,57}$ In the heterogeneous case the stretching of $I(Q, t)$ is due to a superposition of different single exponential relaxations from particles with different relaxation times. On the contrary, in the homogenous case all particles relax identically but nonexponential. Thus, in this case each relaxation process is characterized by the same stretched exponential. In the case of homogeneous dynamics the stretching parameter $\beta_{\mathrm{KWw}}$ is considered as an indicator of the degree of correlation or cooperativity of the relaxation process. Thus, both the homogeneous and the heterogeneous scenarios may give rise to the same stretched KWW function of $I(Q, t)$, but the two scenarios can be distinguished by their different $Q$-dependences of $\left\langle\tau_{p}\right\rangle$. In the heterogeneous case it has been shown ${ }^{56}$ that $\tau_{p}(Q) \propto 1 / Q^{2}$ and in the homogeneous case $\tau_{p} \propto 1 / Q^{2 / \beta_{\mathrm{KWw}}}$, where $\beta_{\mathrm{KWW}}<1$. From our results, based on the assumptions made with approach 1 , we have got values of the shape parameter $\beta_{\mathrm{KWw} p}$ (Table III) that should give an exponent $2 / \beta_{\mathrm{KWW}}>4$ for all samples if the homogeneous scenario was correct. Thus, the data suggest that the dynamic protein processes in all ice-free mixtures are somewhat intrinsically stretched, but that the main reason for the very stretched relaxation function is that it involves a large number of different protein relaxations occurring on different time scales, as discussed above.

It has been shown that it is possible to compare relaxation times measured by dielectric spectroscopy with those measured by neutron scattering at $Q$-values of about $1 \AA^{-1} .^{58}$ Therefore, in Fig. 8 we compare the average relaxation times obtained from the KWW fits at $Q \approx 1 \AA^{-1}$ for both the protein and the solvent in all samples with some of the relaxation processes obtained from dielectric measurement on myoglobin at the hydration level $h=0.5 .^{59}$ The fastest high-temperature process in this figure is due to the $\alpha$-relaxation of the hydration water ${ }^{60}$ and it is fitted by the Vogel-Fulcher-Tamman equation $\langle\tau\rangle=\left\langle\tau_{0}\right\rangle \exp \left[D T_{0} / T-T_{0}\right]$, where $\tau_{0}$ is the relaxation time extrapolated to infinite temperature, which generally is of the order of $10^{-14} \mathrm{~s} . T_{0}$ is the temperature where $\tau$ goes to infinity and the constant $D$ determines the deviation from Arrhenius temperature dependence. From this figure it is obvious that the temperature dependence of the water relaxation times from neutron scattering agrees reasonably well with this dielectric $\alpha$-relaxation. The origin of the slower dielectric relaxation process is not fully clear for the moment but a likely explanation can be that it is related to motions of polar side chains, ${ }^{61}$ possibly together with water molecules bound to the surface. ${ }^{62}$ From Fig. 8 it is also clear that independent of the approach, the temperature dependences of the protein average relaxation times $\left\langle\tau_{p}\right\rangle$ and the corresponding average solvent relaxation times $\left\langle\tau_{s}\right\rangle$ are very similar, thus, supporting the idea that protein fluctuations are "solvent-slaved."11 The temperature dependences are, furthermore, comparable to that of the dielectric process observed on the same time scale. ${ }^{59}$ However, direct comparisons of relaxation times obtained from the present QENS study and dielectric spectroscopy are difficult, mainly due to the fact that the limited experimental energy resolution of the used spectrometer does not allow the protein to be fully relaxed on the experimental time scale.

Finally, it should be noted that our findings for the protein average relaxation times $\left\langle\tau_{p}\right\rangle$ contradict the interpreta- 
tions made in another QENS and dielectric study on protein dynamics. ${ }^{29}$ In that recent study lysozyme hydrated in $\mathrm{D}_{2} \mathrm{O}$ $(h=0.4)$ was used and it was found that their main dielectric process, which here is attributed to water dynamics, was in perfect agreement with the protein relaxation times obtained in their QENS study. Due to this agreement, the authors of Ref. 29 concluded that their main dielectric process was mainly caused by protein dynamics, rather than water dynamics as we suggest here and previously (see, e.g., Refs. 15 and 60). Our interpretation of this dielectric process, i.e., that it is due to the relaxation of water molecules, is supported both by the fact that it is almost universal for a wide range of water containing systems (even "solid" systems where only water molecules can relax on the actual time scale), see, e.g., Ref. 63, and by the fact that the water relaxation process (at temperatures above the melting point) completely relax on the time scale probed in this QENS study, in contrast to the protein dynamics. Moreover, since only a minor fraction of all protein relaxation processes occur on a picosecond time scale (i.e., on the same time scale as hydration water probed by QENS), and the dielectric constant of a protein $(\varepsilon$ $\approx 2-4)$ is very small compared to that of water $(\varepsilon \approx 80$ at room temperature) it is no doubt about that the major contribution to this dielectric relaxation process comes from the relaxation of water.

\section{CONCLUSIONS}

In this study we have explored possible relations between protein and solvent dynamics obtained from QENS. We have also tried to investigate the physical nature of the dynamics of the protein myoglobin and its different water and water:glycerol environments by analyzing the $Q$-dependence of the average relaxation times. However, due to the broad distribution of relaxation times and the relatively narrow time window of the spectrometer, this is evidently a difficult task, which requires that certain assumptions are made. Based on the made assumptions the results suggest that the hydrogen atoms in the protein move in a confined geometry and that the very stretched behavior of the intermediate scattering function $I(Q, t)$ is mainly due to the fact that it involves several protein processes occurring on different time scales, but also that one or several of these dynamical processes are somewhat intrinsically stretched.

In the case of the solvent the results are more conclusive. We found that the $Q$-dependence of the solvent dynamics is well described by the Gaussian jump-length diffusion model, which is commonly used to describe QENS data on both bulk and confined water. The diffusion constants $D$ for the hydration water in samples containing only water are only slightly affected (a factors of $2.0 \pm 0.3$ and $2.6 \pm 0.3$ slower for the hydration levels $h=1$ and $h=0.5$, respectively) compared to bulk water.

Finally, we found that the protein dynamics is strongly related to the solvent dynamics, in agreement with the solvent-slaving idea proposed in, e.g., Ref. 11, but that the protein dynamics does not only depend on the time scale of the solvent dynamics, but also on the amount of solvent. Thus, the protein dynamics is more strongly dependent on the total solvent content than the dynamics of the solvent itself. This implies that the protein dynamics (and therefore also protein activity) can be slowed down, or even prevented, by reducing either the temperature or the amount of solvent or increasing the viscosity of the solvent.

${ }^{1}$ J. A. Rupley, E. Gratton, and G. Careri, Trends Biochem. Sci. 8, 18 (1983).

${ }^{2}$ P. A. Lind, R. M. Daniel, C. Monk, and R. V. Dunn, Biochim. Biophys. Acta 1702, 103 (2004)

${ }^{3}$ J. Fitter, S. A. W. Verclas, R. E. Lechner, H. Seelert, and N. A. Dencher, FEBS Lett. 433, 321 (1998)

${ }^{4}$ F. X. Yang and A. J. Russell, Biotechnol. Bioeng. 49, 709 (1996).

${ }^{5}$ E. Stevens and L. Stevens, Biochem. J. 179, 161 (1979).

${ }^{6}$ J. A. Rupley and G. Careri, Adv. Protein Chem. 41, 37 (1991).

${ }^{7}$ J. A. Rupley, P. H. Yang, and G. Tollin, in Water in Polymers, edited by

S. P. Rowland (American Chemical Society, Washington, D.C., 1980).

${ }^{8}$ V. P. Denisov and B. Halle, Faraday Discuss. 103, 227 (1996).

${ }^{9}$ S. Dellerue and M. C. Bellissent-Funel, Chem. Phys. 258, 315 (2000).

${ }^{10}$ H. D. Middendorf, Physica B 226, 113 (1996).

${ }^{11}$ P. W. Fenimore, H. Frauenfelder, B. H. McMahon, and R. D. Young, Proc. Natl. Acad. Sci. U.S.A. 101, 14408 (2004).

${ }^{12}$ W. Doster and M. Settles, Biochim. Biophys. Acta 1749, 173 (2005).

${ }^{13}$ D. Vitkup, D. Ringe, G. A. Petsko, and M. Karplus, Nat. Struct. Biol. 7, 34 (2000).

${ }^{14}$ M. Tarek and D. J. Tobias, Phys. Rev. Lett. 88, 138101 (2002).

${ }^{15}$ J. Swenson, H. Jansson, J. Hedström, and R. Bergman, J. Phys.: Condens. Matter 19, 205109 (2007).

${ }^{16}$ G. Caliskan, A. Kisliuk, and A. P. Sokolov, J. Non-Cryst. Solids 307, 868 (2002).

${ }^{17}$ R. M. Daniel, J. L. Finney, V. Reat, R. Dunn, M. Ferrand, and J. C. Smith, Biophys. J. 77, 2184 (1999).

${ }^{18}$ J. A. Hayward, J. L. Finney, R. M. Daniel, and J. C. Smith, Biophys. J. 85, 679 (2003).

${ }^{19}$ A. Paciaroni, S. Cinelli, and G. Onori, Biophys. J. 83, 1157 (2002).

${ }^{20}$ J. Fitter, Biophys. J. 76, 1034 (1999).

${ }^{21}$ A. L. Tournier, J. C. Xu, and J. C. Smith, Biophys. J. 85, 1871 (2003).

${ }^{22}$ W. Doster, S. Cusack, and W. Petry, Nature (London) 337, 754 (1989).

${ }^{23}$ J. H. Roh, V. N. Novikov, R. B. Gregory, J. E. Curtis, Z. Chowdhuri, and A. P. Sokolov, Phys. Rev. Lett. 95, 038101 (2005).

${ }^{24}$ A. L. Tournier, V. Reat, R. Dunn, R. Daniel, J. C. Smith, and J. Finney, Phys. Chem. Chem. Phys. 7, 1388 (2005).

${ }^{25}$ E. Cornicchi, G. Onori, and A. Paciaroni, Phys. Rev. Lett. 95, 158104 (2005).

${ }^{26}$ A. M. Tsai, D. A. Neumann, and L. N. Bell, Biophys. J. 79, 2728 (2000).

${ }^{27}$ M. Tarek and D. J. Tobias, Eur. Biophys. J. 37, 701 (2008).

${ }^{28} \mathrm{~J}$. Swenson, H. Jansson, and R. Bergman, in Aspects of Physical Biology: Biological Water, Protein Solutions,Transport and Replication, edited by G. Franzese and M. Rubi (Springer-Verlag, Berlin, 2008), pp. 23-42.

${ }^{29}$ S. Khodadadi, S. Pawlus, J. H. Roh, V. G. Sasaki, E. Mamontov, and A. P. Sokolov, J. Chem. Phys. 128, 195106 (2008).

${ }^{30}$ A. J. Wand, Nat. Struct. Biol. 8, 926 (2001).

${ }^{31}$ L. E. Kay, Nat. Struct. Biol. 5, 513 (1998).

${ }^{32}$ S. Dellerue, A. J. Petrescu, J. C. Smith, and M. C. Bellissent-Funel, Biophys. J. 81, 1666 (2001).

${ }^{33}$ A. Filabozzi, M. Di Bari, A. Deriu, A. DiVenere, C. Andreani, and N. Rosato, J. Phys.: Condens. Matter 17, S3101 (2005).

${ }^{34}$ C. Caronna, F. Natali, and A. Cupane, Biophys. Chem. 116, 219 (2005).

${ }^{35}$ Z. M. Bu, J. Cook, and D. J. E. Callaway, J. Mol. Biol. 312, 865 (2001).

${ }^{36}$ J. Perez, J. M. Zanotti, and D. Durand, Biophys. J. 77, 454 (1999).

${ }^{37}$ C. J. Carlile and M. A. Adams, Physica B 182, 431 (1992).

${ }^{38}$ W. S. Howells, MODES Manual (ISIS Facility, Chilton, U.K., 2003).

${ }^{39}$ R. Giordano, J. Teixeira, and U. Wanderlingh, Physica B 213, 769 (1995).

${ }^{40}$ M. C. BellissentFunel, J. M. Zanotti, and S. H. Chen, Faraday Discuss. 103, 281 (1996).

${ }^{41}$ B. Halle and M. Davidovic, Proc. Natl. Acad. Sci. U.S.A. 100, 12135 (2003).

${ }^{42}$ H. J. Steinhoff, B. Kramm, G. Hess, C. Owerdieck, and A. Redhardt, Biophys. J. 65, 1486 (1993).

${ }^{43}$ M. Marchi, F. Sterpone, and M. Ceccarelli, J. Am. Chem. Soc. 124, 6787 (2002).

${ }^{44}$ E. Y. Lau and V. V. Krishnan, Biophys. Chem. 130, 55 (2007). 
${ }^{45}$ A. Oleinikova, N. Smolin, and I. Brovchenko, Biophys. J. 93, 2986 (2007).

${ }^{46}$ J. Swenson, F. Kargl, P. Berntsen, and C. Svanberg, J. Chem. Phys. 129, 045101 (2008).

${ }^{47}$ H. Jansson, W. S. Howells, and J. Swenson, J. Phys. Chem. B 110, 13786 (2006).

${ }^{48}$ J. Swenson, H. Jansson, W. S. Howells, and S. Longeville, J. Chem. Phys. 122, 084505 (2005).

${ }^{49}$ J. Swenson, R. Bergman, and S. Longeville, J. Chem. Phys. 115, 11299 (2001).

${ }^{50}$ T. Takano, J. Mol. Biol. 110, 537 (1977)

${ }^{51}$ R. V. Rariy and A. M. Klibanov, Proc. Natl. Acad. Sci. U.S.A. 94, 13520 (1997).

${ }^{52}$ R. Sinibaldi, M. G. Ortore, F. Spinozzi, F. Carsughi, H. Frielinghaus, S. Cinelli, G. Onori, and P. Mariani, J. Chem. Phys. 126, 235101 (2007).

${ }^{53}$ M. Tarek and D. J. Tobias, Biophys. J. 79, 3244 (2000).

${ }^{54}$ J. J. Hill, E. Y. Shalaev, and G. Zografi, J. Pharm. Sci. 94, 1636 (2005).

${ }^{55}$ J. A. McCammon, Rep. Prog. Phys. 47, 1 (1984).

${ }^{56}$ A. Arbe, J. Colmenero, M. Monkenbusch, and D. Richter, Phys. Rev.
Lett. 81, 590 (1998).

${ }^{57}$ J. Colmenero, A. Arbe, A. Alegria, M. Monkenbusch, and D. Richter, J. Phys.: Condens. Matter 11, A363 (1999).

${ }^{58}$ A. Arbe, A. Alegria, J. Colmenero, S. Hoffmann, L. Willner, and D. Richter, Macromolecules 32, 7572 (1999).

${ }^{59}$ H. Jansson,R. Bergman, and J. Swenson, "Role of solvent dynamics for protein motions" (unpublished)

${ }^{60}$ J. Swenson, H. Jansson, and R. Bergman, Phys. Rev. Lett. 96, 247802 (2006).

${ }^{61}$ S. Bone, Biochim. Biophys. Acta 916, 128 (1987).

${ }^{62}$ H. Frauenfelder, G. Chen, J. Berendzen, P. W. Fenimore, H. Jansson, B. H. McMahon, I. Mihut-Stroe, J. Swenson, and R. D. Young, Proc. Natl. Acad. Sci. U.S.A. 106(13), 5129 (2009).

${ }^{63}$ S. Cerveny, G. A. Schwartz, R. Bergman, and J. Swenson, Phys. Rev. Lett. 93, 245702 (2004).

${ }^{64}$ J. J. Tuck, P. L. Hall, M. H. B. Hayes, D. K. Ross, and C. Poinsignon, J. Chem. Soc., Faraday Trans. 1 80, 309 (1984).

${ }^{65}$ R. Mills, J. Phys. Chem. 77, 685 (1973).

${ }^{66}$ R. B. Fiorito and R. Meister, J. Chem. Phys. 56, 4605 (1972). 